\title{
High Resolution Mapping of Strains and Rotations \\ using Electron Back Scatter Diffraction
}

\section{Angus J Wilkinson}

Department of Materials, University of Oxford, Parks Road, Oxford OX1 3PH, U.K.

\section{Graham Meaden}

BLG Productions, 3 Sydenham Road, Bristol, BS6 5SH, U.K.

\section{David J Dingley}

Department of Physics, University of Bristol, \& TSL/EDAX Draper, UT 84020, U.S.A.

Author's Version of Manuscript

The published version of record can be found in Materials Science and Technology 22(11):1271-1278 .

OCTOBER 2006 


\section{Abstract}

The angular resolution of EBSD measurements can be significantly improved by using an analysis based on determination of small shifts in features from one pattern to the next using cross-correlation functions. By using pattern shift measurements at many regions of the pattern errors in the best fit strain and rotation tensors can be reduced. We show that elements of the strain tensor (and small misorientations) can be measured to $\pm 10^{-4}$ (and $\pm 0.006^{\circ}$ for rotations).

We apply the technique to two quite different materials systems. Firstly we determine the elastic strain distribution near the interface in a cross-sectioned SiGe epilayer - Si substrate semiconductor heterostructure. Here we use the plane strain boundary conditions at the sample surface to separate every term in the strain tensor.

Secondly we show an application to structural materials by determining the lattice curvature caused by dislocations within the plastic zone associated with the wake and tip of a fatigue crack in a Ni based superalloy. We show that the lattice curvatures can be used to calculate the geometrically necessary dislocation content in the plastic zone. 


\section{Introduction}

Over the last $\sim 10$ years the electron back scatter diffraction (EBSD) technique has expanded from a specialized tool used in a few laboratories across the world to a mainstream materials characterization method used regularly and routinely in the majority of modern materials science laboratories. The rapid uptake of the technique is largely due to the automation of the pattern indexing and orientation measurement procedures coupled with the sophisticated data analysis software that is now commercially available. The wide range of applications of EBSD orientation mapping and quantitative microstructural analysis is testament to the generality built in to the data acquisition and analysis packages.

In this paper we briefly discuss angular resolution of EBSD measurements and its impact on assessment of plastic deformation and elastic strain in materials. We then describe a cross-correlation based analysis of EBSD patterns in which small shifts in pattern features are measured and related to the displacement gradient tensor. We demonstrate that the cross-correlation based method gives an improvement in the angular sensitivity of $\sim 2$ orders of magnitude compared to the usual Hough transform based approach. We illustrate the technique by measuring the elastic strain field (and hence stresses) in a SiGe/Si cross-section sample, and the lattice curvature (and hence GND density distribution) within the plastic zone of a fatigue crack in a superalloy sample. 


\section{Angular Resolution}

The angular resolution of EBSD orientation measurements is often quoted as $1^{\circ}$ to $0.5^{\circ}$, however it is possible to achieve higher resolutions of $\sim 0.1^{\circ}$ by using less binning of the CCD camera during pattern acquisition and smaller bin sizes in the Hough transform accumulator array ${ }^{1}$. Of course there is a consequent reduction in measurement speed.. The $\sim 0.1^{\circ}$ resolution is approximately the angle subtended by a single pixel in the recorded pattern, and appears to be the limit of what can be achieved using the 'conventional' Hough transform based pattern indexing approach. The way by which angular resolution is assessed and quoted for EBSD measurements can be a little deceptive however since a single scalar parameter tends to specified, whereas crystal orientations and indeed misorientations have three degrees of freedom. Wilkinson ${ }^{2}$, and Prior $^{3}$ have demonstrated that for small misorientations the angular uncertainty in individual orientation measurements propagates into a large error in determination of the direction of the misorientation axis. Bate et al ${ }^{4}$ have looked at this problem again recently and have given an analytical relationship for the expected error $\langle\phi\rangle$ in the direction of the misorientation axis as a function of the orientation measurement error $\delta$ and the angle $\theta$ of the misorientation being measured.

$$
\langle\phi\rangle=\arctan \left(\frac{\delta}{\theta}\right)
$$

For a small misorientation of $1^{\circ}$ an orientation measurement error of $1^{\circ}$ causes an error of $\pm 45^{\circ}$ in determining the axis of misorientation so that it is to all intents and purposes unknown. However, reducing the orientation error to $0.1^{\circ}$ allows the misorientation axis to be known to within $\sim \pm 6^{\circ}$. 


\section{Plastic Strain Analysis}

Initial attempts to assess plastic deformation through EBSD were based on the blurring and reduction in contrast of the Kikuchi bands caused by very localized lattice distortions close to dislocations within the diffraction volume ${ }^{5,6}$. Both statistically stored and geometrically necessary dislocations (GNDs) contribute to the pattern blurring, with the GNDs sometimes causing blurring of specific Kikuchi bands, namely those perpendicular to Burger's vectors of the dominant dislocations. ${ }^{7}$. More recent work on plastic deformation has centered on measurement and mapping of the low angle misorientations accumulated within grains as result of deformation. These approaches emphasize the GND content of the dislocation microstructure since statistically stored dislocations produce no long range strain or curvature of the lattice and so do not contribute as strongly to the misorientation measurements. In some works the main aim has been to visualize the spatial distribution of GNDs by imaging the distribution of localized misorientations within individual grains ${ }^{8,9}$, while in others the goal has been to develop some metric that can quantify the GND content averaged over many grains so as to provide a tool for assessing the level of cold work in a component ${ }^{10,11,12}$. A plastic strain sensitivity of $\sim 0.5-1 \%$ has been achieved which is similar to that obtained through assessment of blurring in individual EBSD patterns. The most direct link to GND content is to use EBSD based measurements of lattice curvature to determine dislocation density using Nye's ${ }^{13}$ dislocation tensor. Adams and co-workers ${ }^{14,15}$ have attempted such measurements in $\mathrm{Al}$ bi-crystals and polycrystals, however, scatter in the orientation measurements 
had a large impact on the analysis. This is because the dislocation density is proportional to the gradient in misorientation and differentiation tends to emphasize the noise in the EBSD measurements. In fact el Dasher et al ${ }^{15}$ used a considerable amount of averaging to visualize the pile up of dislocations at the grain boundary during deformation of the Al bi-crystal they studied. Zaefferer et al $^{16}$ have also looked at dislocation pile-up near three different boundaries in Al bi-crystals during plane strain compression, and again had to make use of considerable data averaging to make the near boundary lattice curvature visible. Improvements in misorientations measurements would make dislocation tensor analysis a more realistic proposition.

\section{Elastic Strain Analysis}

Strain measurement has previously proved difficult to achieve with EBSD.

Direct determination of lattice plane spacing through measurement of the Bragg angle can be attempted but is of limited precision estimated at $\sim 5 \times 10^{-3}$ by Wilkinson ${ }^{17}$. This is due to a lack of high order Kikuchi lines (compared say to convergent beam electron diffraction in the TEM) combined with uncertainty in where exactly the Bragg condition falls in the bright-dark intensity profile at the Kikuchi band edge. Recently Keller et al ${ }^{18}$ attempted such measurements in which they compared the lattice parameters of GaAs and GaP and quoted errors of $\pm 2 \times 10^{-3}$.

A more attractive approach is to compare a series of EBSD patterns so as to determine the small shifts in positions of major features (eg zone axes) 
caused by variations in the elastic strains within the sample ${ }^{17,19,20}$. Early work achieved the necessary angular resolution by withdrawing the EBSD detector to a long camera length and hence small capture angle. However, more recently the authors have achieved similar levels of strain sensitivity (i.e. $\pm 2 \times 10^{-4}$ ) with the scintillator screen in its usual large capture angle position ${ }^{21}$. As we will describe in the next section this has the considerable advantage that the entire displacement gradient tensor, containing both strain and rotation information, can be obtained from analysis of each pattern. Bate et $\mathrm{al}^{4}$ have used essentially the same method to determine the rotational part of the displacement gradient tensor (ignoring strains) within grains of deformed and recovered Al samples. Tao and Eades ${ }^{22}$ have used the cross-correlation method to look for small shifts in the position of peaks within Hough transforms corresponding to bands in the original EBSD patterns, though they did not relate these shifts to strains and rotations within the sample. The current authors have also examined this method and can find no advantage in it over direct comparison of the EBSD patterns, however, disadvantages are apparent; namely, increased computation time, and slightly reduced sensitivity to shifts along the $\theta$ (inclination) axis of the Hough transform.

\section{Determining Strains and Small Rotations from EBSD Patterns}

The EBSD measurements were made in the JEOL JSM6500F FEG SEM. EBSD patterns were acquired using a Digiview 12 camera which is a 1300x1030 pixel, Peltier cooled CCD camera with intensities digitized to 12 bits. The phosphor recording screen was held at the usual position so as to 
subtend a capture angle of $\sim 70^{\circ}$ at the sample. TSL/EDAX OIM DC 4 software was used to acquire either a series of patterns in line scans or as regular 2 dimensional arrays (maps). The patterns were analyzed through the use of the Hough transform within the OIM DC software to determine crystal orientation in the normal manner, and were also recorded at full resolution on a hard disk for subsequent off-line analysis to determine strain and small rotations. The latter analysis was carried out using specially developed software by BLG Productions and goes under the title 'CrossCourt'. Exposure times were typically $\sim 2$ seconds.

One pattern in the sequence is taken as a reference pattern and the strain variations and lattice rotations are all measured relative to this point. $A$ number of square regions of interest (ROIs) are distributed over the patterns. For each $\mathrm{ROI}$ the cross correlation between the test pattern and the reference pattern is calculated using fast Fourier transforms. The location of the peak in the cross correlation is determined and gives the shift vector for this ROI. The shift measured is the average of all pixels within the ROI. We assume that the shift measured in this way is equal to the shift at the point at the centre of the ROI. Similarly we assume that the measured shifts are some average value detemined by the strain within the sample volume in the specimen. The shifts ( $x$ and $y$ components) at the centre of each ROI can be related to components of the displacement gradient tensor a using linear equations given in ref 21 . The displacement gradient tensor $\mathbf{a}$ is defined as 


$$
\mathbf{a}=\left(\begin{array}{lll}
\frac{\partial u_{1}}{\partial x_{1}} & \frac{\partial u_{1}}{\partial x_{2}} & \frac{\partial u_{1}}{\partial x_{3}} \\
\frac{\partial u_{2}}{\partial x_{1}} & \frac{\partial u_{2}}{\partial x_{2}} & \frac{\partial u_{2}}{\partial x_{3}} \\
\frac{\partial u_{3}}{\partial x_{1}} & \frac{\partial u_{3}}{\partial x_{2}} & \frac{\partial u_{3}}{\partial x_{3}}
\end{array}\right)
$$

where $\mathbf{u}=\left(u_{1}, u_{2}, u_{3}\right)$ is the displacement at position $\mathbf{x}=\left(x_{1}, x_{2}, x_{3}\right)$ within the sample. A simple hydrostatic dilatation of the crystal generates no shift in the EBSD pattern, and so we cannot solve for individual terms on the lead diagonal of $\mathbf{a}$, but instead can determine differences between these terms (eg $\left[\frac{\partial u_{1}}{\partial x_{1}}-\frac{\partial u_{3}}{\partial x_{3}}\right]$ and $\left.\left[\frac{\partial u_{2}}{\partial x_{2}}-\frac{\partial u_{3}}{\partial x_{3}}\right]\right)$. However, we shall show that boundary conditions can subsequently be used to separate these terms.

Measurements of shifts at four ROIs allow an exact solution to be determined for the system of linear equation, giving eight of the nine degrees of freedom within tensor a. If shifts are measured at more than four ROls then the equations can be solved for the 'best' solution in a least square of errors sense using standard matrix methods. The symmetric part of $\mathbf{a}$ is the strain tensor $\mathbf{e}$, and the antisymmetric part the rotation tensor $\mathbf{w}$, so that components of $\mathbf{e}$ and $\mathbf{w}$ are simply found from a using

$$
e_{i j}=\frac{1}{2}\left(\frac{\partial u_{i}}{\partial x_{j}}+\frac{\partial u_{j}}{\partial x_{i}}\right) \quad, \text { and } \quad w_{i j}=\frac{1}{2}\left(\frac{\partial u_{i}}{\partial x_{j}}-\frac{\partial u_{j}}{\partial x_{i}}\right)
$$

\section{Assessment of Sensitivity}

We have conducted various tests to assess the sensitivity of the method, including making repeated measurements of small tilts and rotations applied to single crystal samples using the SEM stage controls. Data from one such 
example is given in figure 1, where 5 sets of EBSD patterns were recorded from a Si single crystal each after applying nominal rotations about the $x_{2}$ axis of $0^{\circ}, 0.1^{\circ}, 0.2^{\circ}, 0.3^{\circ}, 0.4^{\circ}$ and $0.5^{\circ}$. The cross correlation method was then used to determine the displacement gradient tensor for every pattern using in turn each of the $0^{\circ}$ patterns as the reference. Figure 1 shows the mean values of components of $\mathbf{w}$ and $\mathbf{e}$ as a function of nominal applied rotation. The $w_{13}$ term increases in magnitude with nominal rotation, while the other components remain near zero, in agreement with the expected form of the applied rotation. There is a slight increase in the magnitude of $e_{13}$ as the applied rotation is increased but it is always an order of magnitude smaller than for the $w_{13}$ term. Error bars on the $w_{13}$ and $e_{13}$ data points indicate the standard deviation in the distribution of measurements. In this case the shifts were measured at $20 \mathrm{ROI}$ in the patterns, each region being 256 pixels across. In fact there are different levels of scatter observed in the different components of a due to the anisotropic dispersion of the ROls relative to the reference axes. Figure 2 shows the effects of the number of ROls used in the analysis on the scatter in the measurements, and also illustrates that some components of a have larger associated errors than others. Here the ROIs were distributed uniformly in a circle at the edge of the EBSD patterns. There is increasing overlap between the ROI as their number is increased. This data shows that scatter in the results can be reduced by using a larger number of shift measurements however there is only a $\sim 40 \%$ reduction in the standard deviation on increasing the number of ROls from four to twenty, despite the $\sim 5$ fold increase in the analysis time. Using twenty ROls the 
standard deviation in measured components of the displacement gradient tensor is on average $1 \times 10^{-4}$.

\section{Application to SiGe/Si Semiconductor Structure}

We illustrate the new method by using it to obtain the elastic strain distribution in a semiconductor structure namely a $\sim 1 \mu \mathrm{m}$ thick $\mathrm{Si}_{1-\mathrm{x}} \mathrm{Ge}_{\mathrm{x}}$ epilayer grown on (001) Si. The sample was cleaved and then ground and polished so that observations could be made on a (110) cross section perpendicular to the substrate-epilayer interface. The sample was tilted through $60^{\circ}$ toward the EBSD detector with the tilt axis along the substrate-epilayer interface normal. Patterns were recorded every $10 \mathrm{~nm}$ along a line perpendicular to the interface extending across the epilayer and $\sim 4 \mu \mathrm{m}$ into the substrate. Strains and rotations were determined using pattern shifts measured at twenty ROI uniformly distributed around the edge of the EBSD screen. The reference axes used to describe the strain and rotation tensors are $x_{1}$ parallel to the substrate-interface normal, $x_{3}$ parallel the cross-section surface normal, and $x_{2}$ along the intersection of the sample surface and the interface plane. Figure 3 shows the variations of the most significant terms in the strain and rotation tensors, measured relative to a reference taken at the point in the substrate furthest from the interface. The largest term is in fact the rotation $w_{31}$ about the $x_{2}$ axis which increases in magnitude to a peak rotation of $\sim 0.4^{\circ}$ at the interface. This lattice curvature persists a considerable distance into the substrate and is required to accommodate the displacements along the $x_{3}$ 
direction caused by the relief of the stress within the epilayer at the sample surface. A small localized peak is seen in the $e_{31}$ shear strain variation at the substrate-epilayer interface. In general we would expect this term to be zero or close to it due to the equilibrium condition that the corresponding shear stress $\tau_{31}$ be zero at the free surface. The observed local deviation from zero is a consequence of a very intense shear strain gradient at the interface between the substrate and the misfitting epilayer as has been shown for example in the finite element analysis of Chen and $\mathrm{Li}^{23}$.

The other two terms that come directly from the cross-correlation analysis of the EBSD patterns are the $e_{11}-e_{33}$ and $e_{11}-e_{33}$ variations shown in figure 3. EBSD patterns come from very close to the sample surface where it is reasonable to assume that conditions of plane stress are met. The normal stress $\sigma_{33}$ perpendicular to the free surface must therefore go to zero so we can in general write

$$
\sigma_{33}=0=C_{33 \mathrm{kl}} e_{\mathrm{kl}}
$$

where $C_{\mathrm{ijkl}}$ are the elastic constants refered to the sample (not crystallographic) axis system and the usual summation convention is implied. This condition can be used to separate the three normal strains. With the given sample geometry and symmetry equation 3 simplifies to give

$$
\sigma_{33}=0=C_{3333} e_{33}+C_{3311} e_{11}+C_{3322} e_{22}
$$

with $C_{3333}=166 \mathrm{GPa}$ and $C_{3311}=C_{3322}=64 \mathrm{GPa}$ for $\mathrm{Si}$. The result for the separated normal strains is given in figure 4. The curvature of the lattice shown by the variation of $w_{31}$ with distance along $x_{1}$ causes a bending of the lattice consistent with the increasingly compressive $e_{11}$ stress seen as the interface is approached from the substrate side. All the normal strains show a 
rapid change at the substrate-epilayer interface. The $e_{22}$ strain is of particular interest since we expect little relief of the built in misfit strain by the sample sectioning. Indeed $e_{22}$ remains close to zero through most of the substrate but exhibits a marked compression within the epilayer as should be expected given its larger natural lattice parameter. Within the epilayer the normal strains $e_{11}$ and $e_{33}$ are both tensile due to Poisson expansion associated with the compression along the $x_{2}$ axis.

\section{Application to Fatigue Crack Plastic Zones}

To illustrate application of the technique to a structural material problem we have measured the lattice curvature across the plastic zone associated with the wake and crack tip of a fatigue crack grown in a nickel based superalloy (SRR99). The crack was grown at a temperature of $650^{\circ} \mathrm{C}$, in air at a load ratio of 0.1 . The crack was grown on the (010) plane and in the [100] direction for which crack propagation behaviour has been reported by Henderson and Martin ${ }^{24}$. For the last $\sim 1 \mathrm{~mm}$ of the crack propagation load shedding was used to maintain a stress intensity factor range $\Delta \mathrm{K}$ of $23 \mathrm{MPa} \sqrt{\mathrm{m}}$, which corresponds to a crack growth rate of $\sim 0.6 \mu \mathrm{m}$ per cycle, and the test then terminated. The compact tension test piece was then sectioned so that observation could be made on the mid-plane.

A linescan with EBSD patterns recorded every $100 \mathrm{~nm}$ was taken perpendicular to the crack, that is along the $x_{2}$ direction $\sim 200 \mu \mathrm{m}$ behind the crack tip so as to run across the plastic wake on one side of the crack, see inset to figure 6 . Figure 5 compares the misorientation profile determined 
using the usual Hough transform based analysis with that obtained using the cross correlation based analysis. The general trends and magnitudes obtained from the two methods are comparable. However it is clear that there is much less noise associated with the cross correlation analysis. The crosscorrelation derived misorientation profile indicates that the plastic zone extends $\sim 23 \mu \mathrm{m}$ from the crack. Very close to the crack the patterns were too diffuse and low in contrast to make reliable measurements by either method. Given the symmetric distribution of slip systems with respect to the crack virtually constant lattice rotation would be expected while moving along lines parallel to the crack growth direction (until the crack tip is approached) or along the crack front direction (within plane strain conditions). Thus the only contributions to the dislocation tensor come from the lattice curvature arising from the variation of lattice orientation with distance from the crack plane (ie along the $x_{2}$ axis). Figure 6 shows the variation of different components $\left(w_{\mathrm{ij}}\right)$ of the rotation tensor along the scan line. Note that the Hough transform based data cannot usefully be split into different components since for these small rotations there is far too much scatter in the misorientation axes. The gradients of the curves in figure 6 give the lattice curvature, specifically the variation of $w_{12}$ and $w_{23}$ lead to bending, while the gradient of the $w_{31}$ component describes a twist in the lattice about the normal to the fracture plane. This lattice curvature can be linked to the geometrically necessary dislocation distribution within the plastic wake of the crack using the analysis given by Nye ${ }^{13}$. In the current analysis we simply decompose the dislocation tensor as defined by Nye into a set of pure screw and pure edge dislocations with line and Burgers vectors aligned with the reference axes describing the 
crack geometry. The three terms of the leading diagonal of the tensor, $\rho_{11}$, $\rho_{22}, \rho_{33}$, represent pure screw dislocations and the six cross terms represent the six possible types of pure edge dislocations. The first subscript refers to the direction of the Burgers vector and the second to the direction of the dislocation line. The $w_{12}$ variation is then directly and uniquely linked to the dislocation tensor density component $\rho_{23}$ of edge type GNDs with Burgers vector along $x_{2}$, and line direction along $x_{3}$. Similarly the $w_{23}$ variation yields the density $\rho_{21}$ of edge type GNDs with Burgers vector along $x_{2}$, and line direction along $x_{1}$. Finally the lattice twisting described by $w_{31}$ leads to equal densities $\rho_{11}$ and $\rho_{33}$ of screw GNDs with Burgers vector along $x_{1}$ and $x_{3}$ respectively. The variation of these GND densities is shown in figure 7, in which a negative sign on the dislocation density simply indicates that dislocations with Burgers vectors along the negative direction of the respective Cartesian axes are required to generate the required lattice curvature. Outside the plastic wake the dislocation density fluctuates due to noise on the rotation measurements and has a standard deviation of $\sim 1 \times 10^{12} \mathrm{~m}^{-2}$. The largest term is seen to be $\rho_{23}$ which close to the crack plane has magnitude $\sim-9 \times 10^{13} \mathrm{~m}^{-2}$ increasing steadily to a peak of $\sim+4 \times 10^{13} \mathrm{~m}^{-2}$ at a distance of $\sim 6$ microns from the crack tip. The point at which $\rho_{23}$ changes sign demarks the edge of the cyclic plastic zone which is thus seen to extend by no more than $\sim 5 \mu \mathrm{m}$ from the crack plane. The cyclic plastic zone is thus approximately $1 / 5^{\text {th }}$ the size of the surrounding monotonic tensile plastic zone size which is in excellent agreement with simple estimates of the plastic zone sizes. 
A two dimensional map was also acquired at the tip of the fatigue crack with patterns recorded on a $1 \mu \mathrm{m}$ pitch square grid. Figure 8 compares results obtained with using the conventional Hough transform analysis with those for the new cross correlation based method. There is broad agreement between the spatial distribution of local maxima and minima in the two data sets, though the cross-correlation analysis is clearly much less noisy. Figure $8 a$ shows results of the conventional analysis in which the greyscale indicates disorientation from the exact $(001)[100]$ orientation of the sample, with $5^{\circ}$ (87 mrads) black and $8^{\circ}$ (140 mrads) white. It is possible to visualise the size and shape of the plastic zone in this image. The image is however noisy even though no attempt has been made to represent the axes of misorientation. The better quality of the data obtained using the cross correlation based analysis is illustrated in figure $8 \mathrm{~b}$ which shows a map of a specific component of the rotation field $w_{12}$ (ie the in plane rotations). The measured rotations are approximately anti-symmetric across the crack plane, as should be expected from fracture mechanics theory. The plastic zone appears somewhat wider on the upper side of the crack, and has similar width to that seen in the line scan. Again in accord with the line scan data $w_{12}$ increases in magnitude to a peak approximately $5 \mu \mathrm{m}$ out from the crack plane and then decreases again. This peak value appears to indicate the position of the outer edge of the cyclic plastic zone.

\section{Conclusions}

1. We have shown that it is possible to measure small displacements in local regions of a wide angle EBSD pattern using a cross 
correlation function where the distorted pattern is compared with an undistorted reference pattern. These measurements can be interpreted to yield the local strain tensor within the sample volume of the specimen. When our cross correlation analysis is used to determine small pattern shifts at twenty uniformly distributed regions at the edge of wide angle EBSD patterns we estimate that terms in the strain and rotation tensor can be determined to $\pm 10^{-4}$. This corresponds to a misorientation sensitivity of $\pm 0.006^{\circ}$.

2. In a cross sectioned SiGe/Si heterostructure EBSD measurements showed an in plane compression in the epilayer, while out of the cross section plane stresses were relaxed leading to a slight tensile strain through Poisson ratio effects. The expansion of the epilayer is accommodated by an elastic curvature near the surface of the cross section which extends a considerable distance into the substrate from the interface.

3. In the plastic wake associated with a fatigue crack in a Ni based superalloy EBSD measurements showed lattice rotations about each of the three Cartesian axes. Rotations were largest about an axes parallel to the crack front. The types and densities geometrically necessary dislocations were calculated from the observed lattice curvature. The sense of the lattice curvature and hence sign of the dislocations was reversed on going from the outer tensile plastic zone to the inner cyclic plastic zone. 



\section{References}

${ }^{1}$ Humphreys FJ, Huang Y, Brough I, Harris C , J. Microscopy, 195, 212-216 (1999)

${ }^{2}$ A. J. Wilkinson, Scripta Mater. , 44, 2379-2385, (2001)

${ }^{3}$ D.J. Prior, J. Microscopy, 195, 217-225 (1999)

${ }^{4}$ P.S. Bate, R.D. Knutsen, I. Brough and F.J. Humphreys, J. Microscopy, 220, 36-46, (2005)

${ }^{5}$ P N Quested, P J Henderson, \& M Mclean, Acta Metall., 36, 2743-2752, (1988)

${ }^{6}$ A. J. Wilkinson \& D. J. Dingley, Acta Met. et Mater., 39, 3047-3055, (1991)

${ }^{7}$ A. J. Wilkinson, Proc. EMAG'91, Inst Phys Conf Series No 119, 197-200 (1991)

${ }^{8}$ D P Field, P B Trivedi, S I Wright, \& M Kumar, Ultramicroscopy, 103, 33-39, (2005)

${ }^{9}$ G. M. Pennock \& M. R. Drury, J Microscopy, 217, 130-137 (2005)

${ }^{10}$ E M LeHockey, Y Lin, and O E Lepik, Mapping residual plastic strain in materials using electron backscatter diffraction, Electron Backscatter

Diffraction in Materials Science, Kluwer Academic /Plenum Publishers (eds A. J. Schwartz et al), pp. 247-264, (2000).

11 J. C. Glez, and J. Driver, J. Appl. Cryst., 34, 280-288 (2001)

12 M Kamaya, A J Wilkinson and J M Titchmarsh Nuclear Engineering \& Design, 235, 713-725 (2005)

13 J Nye, Acta Metall., 1, 153, (1953)

${ }^{14}$ S Sun, B L Adams, \& W E King, Phil. Mag. A, 80, 9-25, (2000)

15 BS El-Dasher, BL Adams, \& AD Rollett, Scripta Mater., 48, 141-145, (2003)

${ }^{16}$ S Zaefferer, JC Kuo, Z Zhao, M Winning, D Raabe, Acta Mater., 51, 47194735, (2003)

${ }^{17}$ A. J. Wilkinson, Mater. Sci. Tech., 13, 79-84 (1997)

${ }^{18}$ R. R. Keller, A. Roshko, R. H. Geiss, K. A. Bertness and T. P. Quinn, Microelectronic Engineering, 75, 96-102, (2004)

${ }^{19}$ K. Z. Troost, P. van der Sluis and D. J. Gravestijn, Appl. Phys. Lett., 62, 1110-1112, (1993)

${ }^{20}$ A J Wilkinson, Ultramicroscopy 62, 237-247, (1996)

${ }^{21}$ A J Wilkinson, G Meaden, and D J Dingley, Ultramicroscopy, in press (2005)

${ }^{22}$ X. Tao and A. Eades, Microscopy and Microanalysis 11, 341-353, (2005)

${ }^{23}$ C R Chen and S X Li, J. Mater. Sci. , 35, 1145-1152, (2000)

${ }^{24}$ M B Henderson and J W Martin, Acta Metall. Mater., 44, 111-126, (1995) 


\section{Figures}

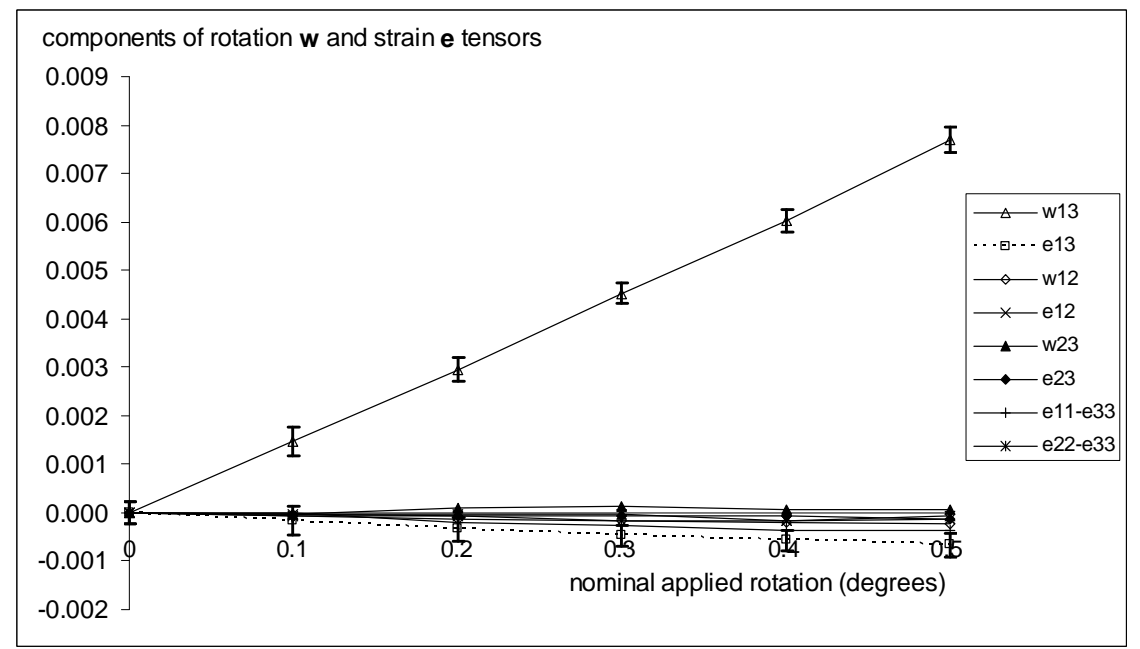

Figure 1: Measured strain and rotation tensor components for rotations applied about the $x_{2}$ axis to a single crystal.

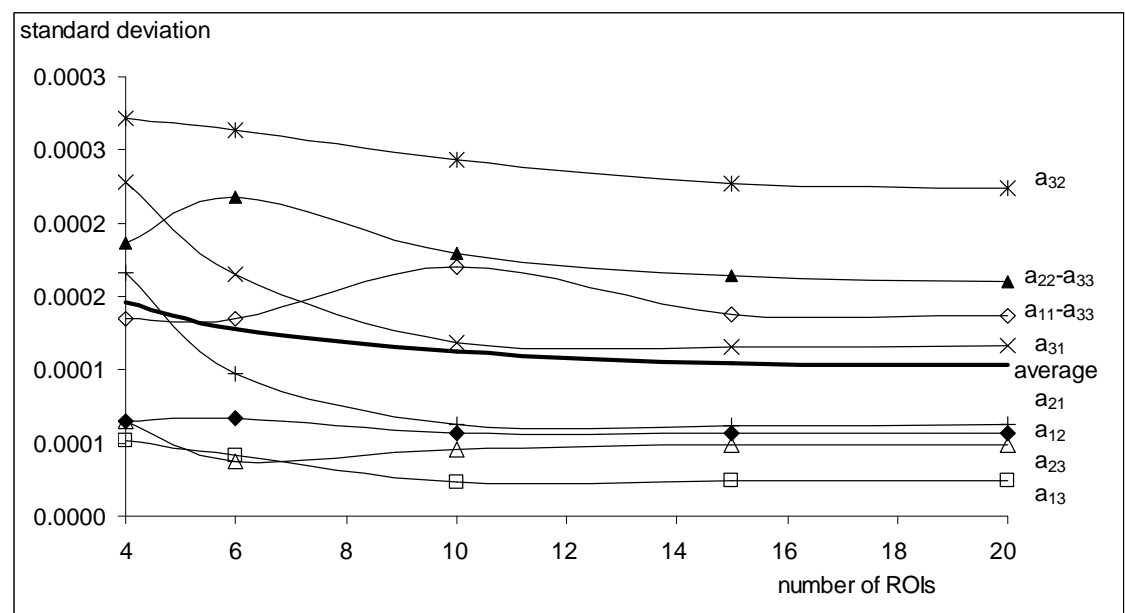

Figure 2: Effect of number of regions of interest (ROIs) on the standard deviation in components of the displacement gradient tensor $a_{\mathrm{ij}}$. 


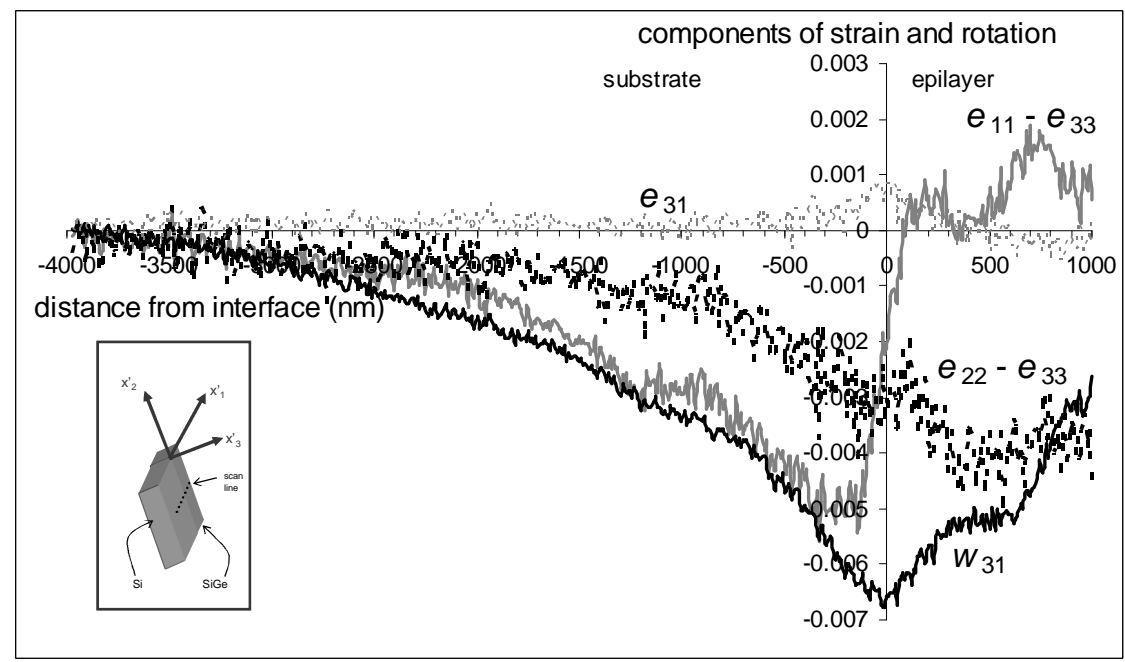

Figure 3: Strain and rotations measured close to the interface between a SiGe epilayer grown on a Si substrate.

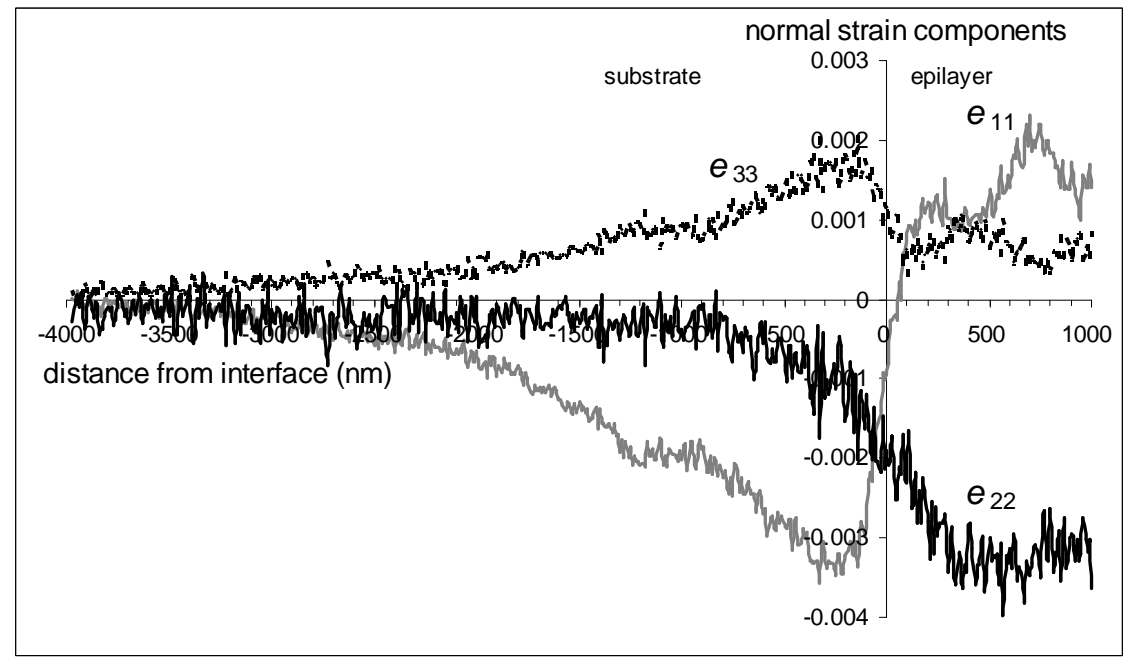

Figure 4: Separation of normal strains in the SiGe/Si cross section. 


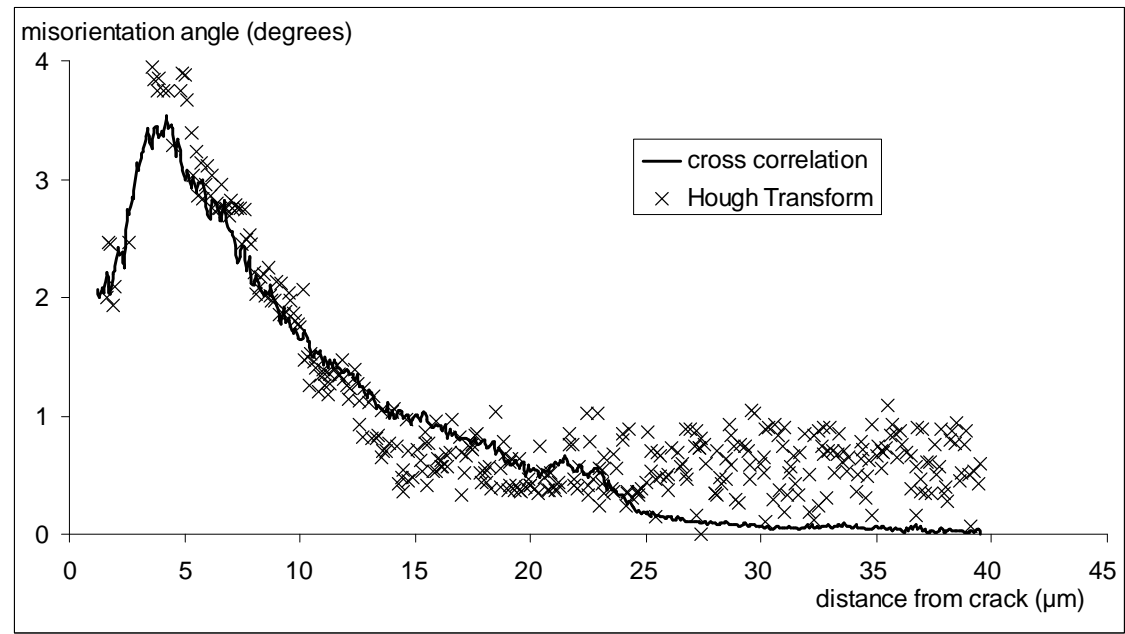

Figure 5: Comparison of lattice rotation determined using conventional Hough transform based orientation measurements with those from cross-correlation analysis. Reference point is furthest crack.

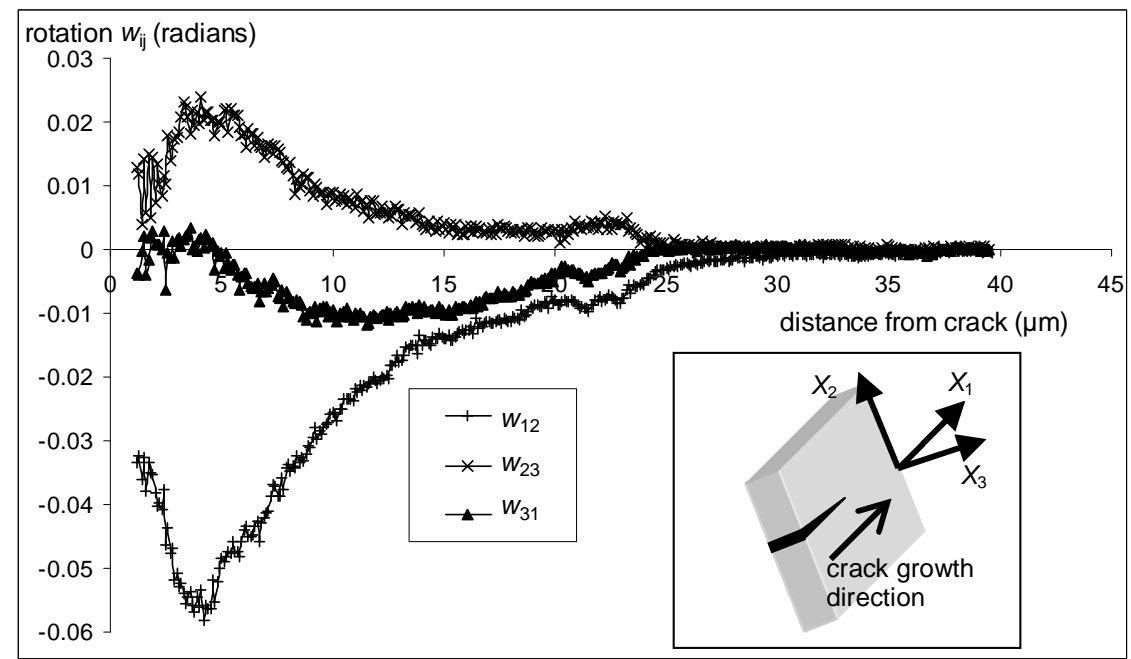

Figure 6: Components of lattice rotation tensor across plastic wake near fatigue crack. 


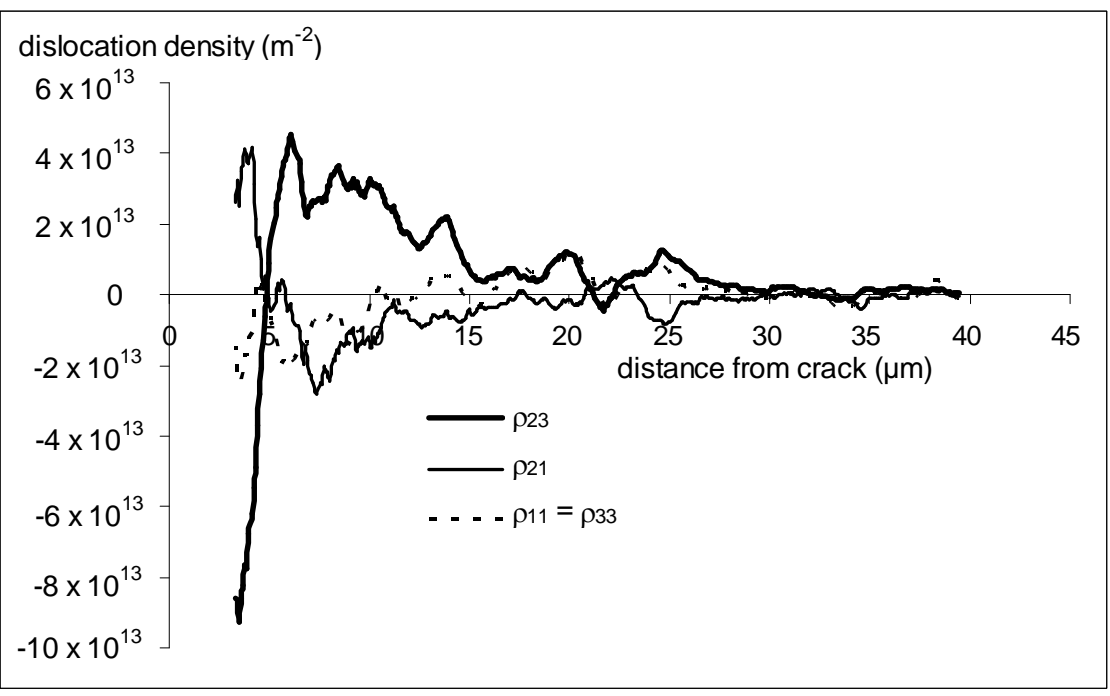

Figure 7: Distribution of geometrically necessary dislocations in plastic wake associated with a fatigue crack. 
(a)

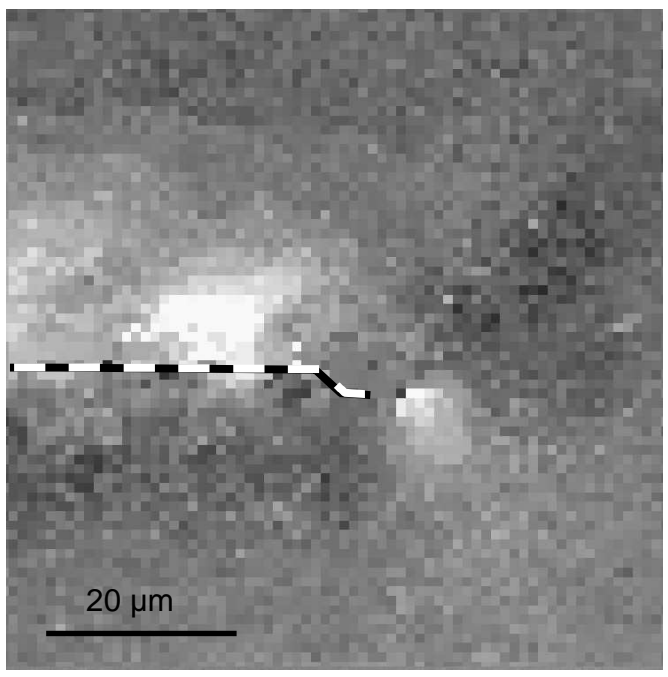

(b)

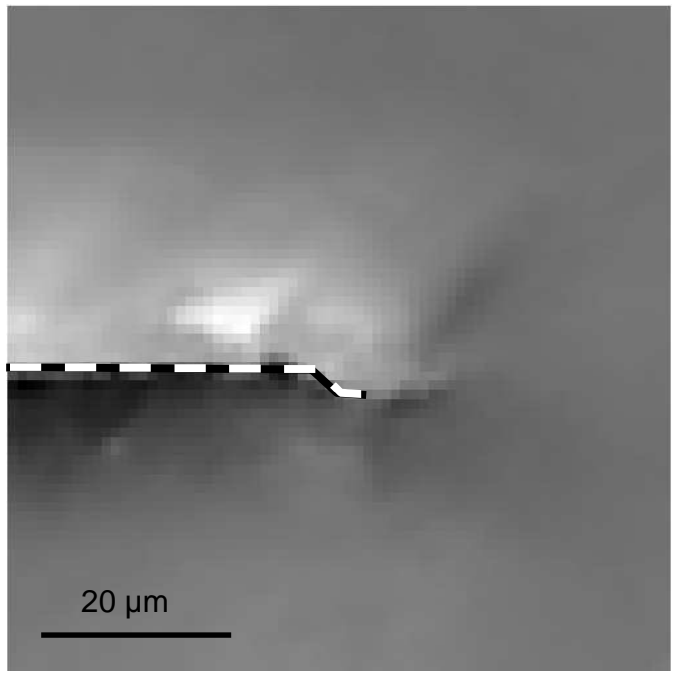

Figure 8: EBSD maps at fatigue crack tip. (a) Hough transform analysis results indicating disorientation from (001)[100] orientation of sample (87 mrads $\rightarrow$ black and 140 mrads $\rightarrow$ white). (b) Cross-correlation analysis results showing $w_{12}$ rotation component (44 mrads $\rightarrow$ black and -53 mrads $\rightarrow$ white). 